\title{
Contribution of recently measured nuclear data to reactor antineutrino energy spectra predictions
}

\author{
M. Fallot ${ }^{1, a}$, S. Cormon ${ }^{1}$, M. Estienne ${ }^{1}$, A. Algora ${ }^{2,3}$, V.M. Bui ${ }^{1}$, A. Cucoanes ${ }^{1}$, M. Elnimr ${ }^{1}$, L. Giot ${ }^{1}$, \\ D. Jordan ${ }^{2}$, J. Martino ${ }^{1}$, A. Onillon ${ }^{1}$, A. Porta ${ }^{1}$, G. Pronost ${ }^{1}$, A. Remoto ${ }^{1}$, J.L. Taín ${ }^{2}$, F. Yermia ${ }^{1}$, \\ and A.-A. Zakari-Issoufou ${ }^{1}$ \\ ${ }^{1}$ SUBATECH, CNRS/IN2P3, Université de Nantes, Ecole des Mines de Nantes, 44307 Nantes, France \\ ${ }^{2}$ IFIC (CSIC-Univ. Valencia), Valencia, Spain \\ ${ }^{3}$ Institute of Nuclear Research, Debrecen, Hungary
}

\begin{abstract}
This paper attempts to summarize the actual problematic of reactor antineutrino energy spectra in the frame of fundamental and applied neutrino physics. Nuclear physics is an important ingredient of reactor antineutrino experiments. These experiments are motivated by neutrino oscillations, i.e. the measure of the $\theta_{13}$ mixing angle. In 2011, after a new computation of the reactor antineutrino energy spectra, based on the conversion of integral data of the beta spectra from ${ }^{235} \mathrm{U}$, and ${ }^{239 ; 241} \mathrm{Pu}$, a deficit of reactor antineutrinos measured by short baseline experiments was pointed out. This is called the "reactor anomaly", a new puzzle in the neutrino physics area. Since then, numerous new experimental neutrino projects have emerged. In parallel, computations of the antineutrino spectra independant from the ILL data would be desirable. One possibility is the use of the summation method, summing all the contributions of the fission product beta decay branches that can be found in nuclear databases. Studies have shown that in order to obtain reliable summation antineutrino energy spectra, new nuclear physics measurements of selected fission product beta decay properties are required. In these proceedings, we will present the computation methods of reactor antineutrino energy spectra and the impact of recent beta decay measurements on summation method spectra. The link of these nuclear physics studies with short baseline line oscillation search will be drawn and new neutrino physics projects at research reactors will be briefly presented.
\end{abstract}

\section{Introduction}

Antineutrinos are emitted in huge quantities by nuclear reactor cores; about $10^{21}$ antineutrinos per second for a $1 \mathrm{GWe}$ power plant. This feature has triggered numerous particle physics experiments at reactors since decades, the first being the one performed by Reines and Cowan at the Savannah River reactor in 1956 [1], giving the first experimental evidence of the neutrino existence. In the recent years, new generation reactor neutrino experiments were designed in order to measure the last mixing angle ruling neutrino oscillations, the $\theta_{13}$ mixing angle. These experiments use multi-detectors at reactors, with one or several near detectors placed close to the reactor cores in order to measure the emitted

\footnotetext{
ae-mail: fallot@subatech.in2p3.fr
}

This is an Open Access article distributed under the terms of the Creative Commons Attribution License 2.0, which permits unrestricted use, distribution, and reproduction in any medium, provided the original work is properly cited. 
antineutrino flux before it oscillates, and one or several far detectors at a location chosen to maximize the oscillation probability. The design of the detectors should be identical in order to eliminate most of the systematics. This principle applies to the Double Chooz, Daya Bay and Reno experiments [2-4]. But even with this setup, if the detectors are not placed on the isoflux lines of the reactors of the plant, a correction to the measurements should be applied before comparing the near and far detectors. The knowledge of the antineutrino energy spectra and of the reactor fission rates is then mandatory to correct for this bias. In the case of the Double Chooz experiment, only the far detector has been taking data during the first phase of the experiment. A huge effort was devoted to reactor simulations [5] and to revisit the existing methods to compute the antineutrino energy spectrum emitted by the plant, as a precise prediction is mandatory to compare with the far detector measurements. These new calculations $[6,7]$, based on the conversion of the very precise integral beta spectra measured by Schreckenbach et al. at the ILL reactor [8], have led to a reinterpretation of the results of the short baseline reactor neutrino experiments [9], evidencing a deficit of the measured reactor antineutrino flux w.r.t. the prediction, @ $3 \sigma$. This deficit was called the "reactor anomaly". Among several possible explanations, the potential existence of sterile neutrinos has been evoked [9], triggering numerous new experimental projects aiming at shedding the light on these short baseline oscillations [10]. In this context, an independant evaluation of the antineutrino energy spectra would be necessary, as no other measurement of the integral beta spectra associated to the fissions of ${ }^{235} \mathrm{U}$ and ${ }^{239 ; 241} \mathrm{Pu}$ has been performed up to now. The antineutrino spectra computed with the summation method (SM), relying on the summation of the contributions of the beta decay of all fission products in a reactor core, could constitute a possible independant evaluation.

In the seventies, was born the idea that reactor antineutrino detector could provide a direct image of the fuel content inside the core, as a function of time [11]. While experiments during the eighties evidenced experimentally the relationship between the antineutrino flux and energy spectrum with the fuel content and the power of the reactor [12,13], this idea was only developed lately with the development of dedicated projets worldwide $[14,15]$ and the recent creation of dedicated Working Groups at the IAEA and at the ESARDA Novel Approaches/Novel Technologies WG [14-16]. It is mandatory in this field to be able to predict the antineutrino emission characteristics associated to various reactor designs [17]. The SM is the only predictive method that could allow such calculations. Antineutrino detection for reactor monitoring is thus another motivation to improve the quality of the beta decay properties stored in the evaluated databases for fission products.

It has been shown that new measurements of fission product beta decay properties were necessary in order to obtain reliable SM antineutrino energy spectra with error bars comparable with the converted spectra from the integral measurements $[6,18]$. For all these motivations, a new experimental campaign has been started by the Total Absorption Spectroscopy (TAS) [19] collaboration in order to measure with the TAS technique beta decay properties of a selected set of nuclei selected thanks to antineutrino spectra simulations. In a first section, we will present briefly the two computation methods of reactor antineutrino energy spectra and the impact of the latest published TAS data on antineutrino energy spectra. The second section will be devoted to the reactor anomaly and the brief presentation of projects of neutrino experiments at research reactors.

\section{Computation methods of reactor antineutrino energy spectra and recent results}

\subsection{The conversion method (CM)}

The first attempts to re-compute reactor antineutrino energy spectra relied on the summation technique, taking advantage of the huge information present nowadays in the nuclear databases [6]. 
The obtained beta energy spectra for the main fissible isotopes were compared with the integral beta spectra precisely measured by Schreckenbach's team at the ILL research reactor [8]. The comparison showed an agreement at the $10 \%$ level, which was not sufficient for neutrino physics experiments. The high energy part of the spectra is over-estimated by the SM and some shape distortions are observed. This trend is compatible with the so-called Pandemonium effect [20]. Interested readers can refer to [19] for further explanations. Benefitting from the gathered nuclear data, the CM of the ILL beta integral data was then revisited in order to obtain more precise antineutrino spectra. The conversion procedure consisted originally in fitting the integral beta energy spectra with 30 effective beta branches in order to convert them. The new method used most of the experimental beta branches given by the nuclear databases and fitted $90 \%$ of the ILL beta spectra with them, fitting the $10 \%$ remaining part with 5 effective branches [6]. Thanks to this new method, the correction to the Fermi theory could be applied at the branch level and the real charge $(Z)$ distribution of the fission products could be taken into account, replacing the mean $\mathrm{Z}$ fit that was not accurate enough. These corrections led to a $3 \%$ normalisation shift upward w.r.t. the antineutrino spectra obtained in [8], triggering the "reactor anomaly" quoted above [9]. Huber revisited the CM in parallel and found similar results [7]. Several possible explanations can be invoked to explain the newly observed deficit of reactor antineutrinos at experiments placed closer than $100 \mathrm{~m}$ away from reactor cores. There is a large uncertainty associated to the weak magnetism term entering in the computation of beta decay spectra, which could change the normalization of the predictions of the detected antineutrino flux if revealed too far from the adopted value [7]. In addition, these converted spectra rely entirely on the normalization of the ILL data, for which no other measurement exist. These data are still nowadays the data of reference for reactor neutrino experiments. The third potential explanation is the presence of short baseline oscillations, with the mixing of the three standard neutrino flavours into one or two sterile neutrinos $[9,10]$. In this context, new measurements or alternative methods to compute the antineutrino energy spectra would be desirable.

\subsection{The summation method (SM) and the impact of the latest TAS measurements on the antineutrino spectra}

The anti-v spectrum associated with one of the 4 fissioning isotopes in a moderated reactor can be computed as the sum of the contributions of all fission products thanks to the use of the full information available per nucleus in Nuclear Databases (NDB). The $\beta / \bar{\nu}$ spectrum per fission of a fissible isotope $S_{k}(E)$ can be broken-up into the sum of all fission product $\beta / \bar{v}$ spectra weighted by their activity $A_{f p}$

$$
S_{k}(E)=\sum_{f p=1}^{N_{f p}} A_{f p} \times S_{f p}^{b}\left(Z_{f p}, A_{f p}, E_{0 f p}^{b}, E\right) .
$$

Eventually, the $\beta / \bar{\nu}$ spectrum of one fission product is the sum over the b branches of all $\beta$ decay spectra (or associated $\bar{v}$ spectra), $S_{f p}^{b}$ (in Eq. (1)), of the parent nucleus to the daughter nucleus weighted by their respective branching ratios. To calculate these quantities, we used the MCNP Utility for Reactor Evolution (MURE) code [17,21] to extract the fission rates and the fission product activities as a function of time. The Fermi theory has been implemented in MURE in order to calculate the $\beta / \bar{v}$ spectrum, $S_{f p}^{b}$, of each $\mathrm{b}$ branch of the $\mathrm{fp}^{\text {th }}$ fission product. $B R_{f p}^{b}, Z_{f p}, A_{f p}$ and $E_{0 f p}^{b}$ have been extracted from the selected NDB. The $\beta$ spectrum $S_{f p}^{b}$ in Eq. (1) is derived from the Fermi theory taking into account Huber's prescriptions for the treatment of corrections to be applied to the calculation [7].

The last ingredient required to compute our calculation with the SM are nuclear data bases which contain all the nuclei information. We have determined a selected choice of NDB. Details can be found in $[18,22]$. The choice was carefully made in order to avoid as much as possible Pandemonium-biased 


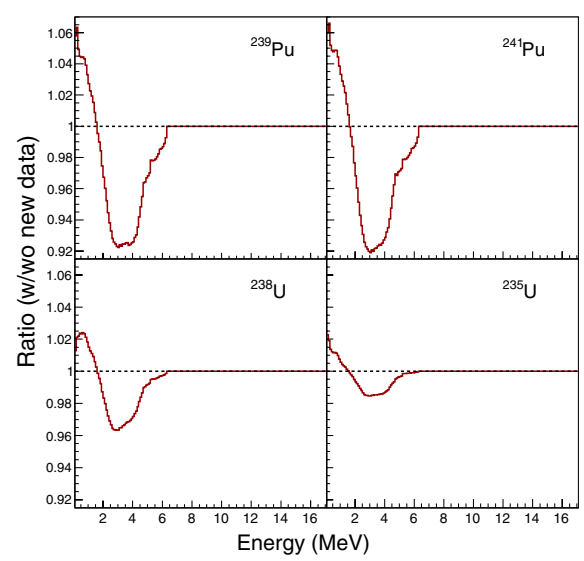

Figure 1. Ratios of the new predicted spectra obtained with and without the last TAS data presented in [23] and extracted from [18].

data. The Total Absorption Spectroscopy technique is an alternative to the use of Ge detectors allowing to circumvent the Pandemonium effect. The author refer to Z. Issoufou's contribution to this conference for more details about the Pandemonium effect, and the TAS technique [19].

The final calculated spectra obtained with the SM for ${ }^{235 ; 238} \mathrm{U}$ and ${ }^{239 ; 241} \mathrm{Pu}$ with $100 \mathrm{keV}$ bins and with the last TAS data can be found in [18]. They are the last up-to-date predictions for these spectra which offer a more precise shape than before [8]. A comparison with the last Huber's computations over the range 2 to $8 \mathrm{MeV}$ has also been proposed. It shows reasonable agreement in the normalization and shape. We reach at maximum $10 \%$ discrepancy up to $7 \mathrm{MeV}$.

In Figure 1, the ratio of the new predicted spectra and the ones obtained with the same data set but the latest TAS data for ${ }^{102 ; 104 ; 105 ; 106 ; 107} \mathrm{Tc},{ }^{101} \mathrm{Nb}$ and ${ }^{105}$ Mo published in [23], previously simulated with JEFF are presented and illustrate how strongly the Pandemonium effect affects the global anti- $v$ energy spectra of ${ }^{235 ; 238} \mathrm{U}$ and ${ }^{239 ; 241} \mathrm{Pu}$. A noticeable deviation from unity (maximum $8 \%$ decrease) is obtained for ${ }^{239 ; 241} \mathrm{Pu}$. We observe a maximum of $3.5 \%$ deviation for ${ }^{238} \mathrm{U}$. For ${ }^{235} \mathrm{U}$, as these nuclei have a small contribution to its spectrum, the effect is smaller than $1.5 \%$ at $\sim 3 \mathrm{MeV}$. Moreover, by convolution with the detection cross section (inverse beta decay), the resulting $\bar{v}$ flux corresponding to pure fissions of ${ }^{235} \mathrm{U},{ }^{239} \mathrm{Pu},{ }^{241} \mathrm{Pu}$ and ${ }^{238} \mathrm{U}$ is $99.1 \%, 94.53 \%, 94.76 \%$ and $98.09 \%$ respectively relative to previously obtained flux. It stresses again the importance of the Pandemonium effect and the necessity to multiply TAS data measurements to improve the predictiveness of the method.

\section{The reactor anomaly and short baseline experiments}

The search for sterile neutrinos has started with the LSND results [24], showing an excess of electronic neutrinos events in a flux of neutrinos from stopped pion decay.

The MiniBooNE experiment attempted to perform another measurement at the same L over E ratio [25] and an excess of events in appearance neutrino and antineutrino spectra was found, which could be consistently interpreted in terms of oscillations. The GALLEX [26] and SAGE [27] solar neutrino experiments reported also a deficit in the awaited neutrino flux obtained with intense artificial ${ }^{51} \mathrm{Cr}$ and ${ }^{37} \mathrm{Ar}$ calibration source measurements [28], with a significance of $2.9 \sigma$. The most recent anomaly comes from the reactor sector [9]. The discrepancy between the predicted flux and the measurements 
arises not only from the newly converted spectra, but also from the revision of the neutron life-time and the contribution of off-equilibrium effects (corrections due to long-lived fission product contributions), leading to a deficit with a significance of $3 \sigma$. The reactor anomaly points toward a sterile neutrino search of $\Delta m^{2} \approx 1 \mathrm{eV}^{2}$ to account for a rate reduction at $L \approx 10-100 \mathrm{~m}$.

In order to interprete all these data consistently, several neutrino schemes can be contemplated; 3 standard neutrino flavours plus one or two sterile neutrinos for instance, the ordering depending on the mass hierarchy assumed for the sterile neutrino(s) w.r.t. the 3 other ones (see for instance [29]. Eventually, the latest global analysis, trying to find a common explanation to all the experimental results in terms of oscillations encounters difficulties to cope with both appearance and disappearance data. The best compatibility is found for the $1+3+1$ scheme with a p-value of $2 \%$ and worse compatibilities in the $3+1$ and $3+2$ schemes [30]. The case of two sterile neutrinos is thus the one which could remain compatible with the experimental results. Nevertheless the existence of two sterile neutrinos is now difficult to reconcile with the latest Planck results [31] giving a number of relativistic degrees of freedom $\mathrm{N}_{\text {eff }}$ of $3.62^{+0.50}-0.48 @ 95 \%$ C.L. (highest result depending on the models assumed). However only a direct measurement could really solve the puzzle of short baseline oscillations.

This is the reason why a huge number of projects has emerged lately. Several methods are contemplated, some using diasppearance search or accelerator based search for eV-scale oscillations. We can quote for the latter case the MicroBooNE experiment or the LANL LAr (Liquid Argon) experiment, the LAr-TPC muon spectrometer at CERN SPS or the stored muon beam experiment (nuSTORM). The use of radio-active source experiments next to large detectors has been proposed (see the projects of artificial source placed next to Kamland (Ce-Land), or Borexino, SNO+, Daya Bay detectors, SAGE-2, LENS...). An alternative is to perform reactor experiments at very short baseline and close to a small reactor core, using shape analysis to be independent from the calculated antineutrino spectra. Here again numerous projects have emerged. Without being exhaustive we can quote the SCRAAM, Neutrino4 and STEREO [32] experiments for detectors based on liquid scintillator targets and DANSS and SoLid [33] for detectors based on segmented plastic designs. The Nucifer experiment [34], though not designed for sterile neutrino search may bring some hints after the required upgrade of the shielding of the detector. The authors quote the following reviews $[10,35,36]$ for interested readers to find more complete informations on experimental projects of the field.

\section{Conclusions and outlooks}

In these proceedings, we have presented a contribution of nuclear physics research to particle physics with the study of neutrino fundamental and applied physics using nuclear reactors. Reactor cores are being used to measure neutrino oscillation parameters such as the theta 13 mixing angle or lately to evidence the potential existence of sterile neutrinos which could explain short baseline oscillations. Nuclear structure and data play an important role in the computation of reactor antineutrino energy spectra, at the origin of the "reactor anomaly". Summation method spectra relying on the beta decay data of the fission products could constitute an alternative to the converted antineutrino spectra relying on the unique measurements of integral beta spectra made at the ILL research reactor. It was shown recently that in order to obtain reliable summation method antineutrino predictions, one should correct the nuclear databases from the Pandemonium effect for a list of selected fission products [18]. To circumvent the Pandemonium problem, the Total Absorption Spectroscopy technique could be employed, and an experimental campaign has started [19]. In parallel, numerous projects in the neutrino physics field have emerged in order to evidence the potential existence of sterile neutrinos, that could explain the short baseline deficit seen at reactors. First answers to this puzzle coming from direct measurements could be obtained at a 3 to 5 year scale from now. 


\section{References}

[1] Frederick Reines and Clyde L. Cowan, Jr., Nature 178, (1956) 446

[2] Y. Abe et al. (Double Chooz collaboration), Phys. Rev. Lett. 108, 131801, (2012)

[3] F. P. An et al., Phys. Rev. Lett. 108 (2012) 171803

[4] J. K. Ahn et al., Phys. Rev. Lett. 108 (2012) 191802

[5] A. Onillon, on behalf of the Double Chooz experiment, Proc. of the International Nuclear Data conference (2013) New York, US

[6] Th. Mueller et al., Phys. Rev. C 83, 054615 (2011)

[7] P. Huber, Phys. Rev. C 84, 024617 (2011)

[8] A. A. Hahn et al., Phys. Lett. B218, 365 (1989) and references therein

[9] G. Mention et al., Phys. Rev. D83, 073006 (2011)

[10] K. N. Abazajian et al., http://arxiv.org/abs/1204.5379

[11] L.A. Mikaelian, 1977, Proc. Int. Conf. Neutrino-77, v.2, p.383

[12] Klimov, Yu.V., Kopeikin, V.I. . Rev, Mikaélyan, L.A., et al., Atomic Energy, 76, 123-127 (1994)

[13] Y. Declais et al., Nucl. Phys. B434, 503 (1995)

[14] M. Fallot, Proc. of the 35rd ESARDA annual meeting, Brugge, Belgium (2013)

[15] M. Fallot, Proc. of the International Nuclear Data conference, (2013) New York, US

[16] IAEA Report SG-EQGNRL-RP-0002 (2012) and IAEA Report STR-361, (2009)

[17] S. Cormon et al., Proc. of the International Nuclear Data conference, (2013) New York, US

[18] M. Fallot et al., Phys. Rev. Lett. 109, 202504 (2012)

[19] A.-A. Zakari-Issoufou et al., contribution to these conference proceedings (2013)

[20] J. C. Hardy et al., Phys. Lett. B71, (1977) 307

[21] MURE, http://www.nea.fr/tools/abstract/detail/nea-1845. O. Meplan, Tech. Rep. LPSC 0912 and IPNO-09-01 (2009)

[22] M. Estienne et al., Proc. of the International Nuclear Data conference, (2013) New York, US

[23] A. Algora et al., Phys. Rev. Lett. 105, 202501 (2010)

[24] A. Aguilar et al., LSND collab., Phys. Rev. D64, 112007 (2001)

[25] A.A. Aguilar-Arevalo et al., MiniBooNE Collaboration, arXiv:1303.2588

[26] GALLEX, W. Hampel et al., Phys. Lett. B420, 114 (1998)

[27] SAGE, J. N. Abdurashitov et al., Phys. Rev. C59, 2246 (1999), hep-ph/9803418

[28] C. Giunti, M. Laveder, Y.F. Li, Q.Y. Liu, H.W. Long, Phys. Rev. D86 (2012) 113014

[29] Th. Schwetz, seminar given at the APC laboratory, http://www.apc.univ-paris7.fr/APC_ CS/files/upload/paris-apc.pdf

[30] J. Kopp, P.A.N. Machado, M. Maltoni, T. Schwetz, arXiv:1303.3011

[31] P.A.R. Ade et al., Planck Collaboration, arXiv:1303.5076

[32] A. Stutz, IN2P3 SC on behalf of the STEREO collab., http://csi.in2p3.fr/CRetTransp. html

[33] F. Yermia, IN2P3 SC on behalf of the SoLid collab., http://csi.in2p3.fr/CRetTransp. html

[34] A. Cucoanes on behalf of the Nucifer collab., Proc. of the International Nuclear Data conference, (2013) New York, US

[35] A.P. Serebrov, http://hepd.pnpi.spb.ru/hepd/events/abstract/Serebrov-6.11. 2012.pdf

[36] J.M. Conrad, C.M. Ignarra, G. Karagiorgi, M.H. Shaevitz, and J. Spitz Advances in High Energy Physics Volume 2013, Article ID 163897. http://dx.doi.org/10.1155/2013/163897 